\title{
Historia de la cirugía de cráneo, de los tumores cerebrales y de la epilepsia en México
}

\author{
F. Chico-Ponce de León
}

Universidad Nacional Autónoma de México. Hospital Infantil de México Federico Gómez. Instituto Nacional de Psiquiatría Ramón de la Fuente.

\section{Resumen}

El primer informe impreso sobre una cirugía de tumor intracerebral en el mundo es el realizado por Bennett y Godle, en 1884, en Londres, sobre un glioma. Se publican, en la misma época, intervenciones sobre este tipo de cirugía en Francia, con Chipault, en Italia con Durante, en Estados Unidos con Keen, en Alemania con Krause y Oppenheim, entre otros. En México Rafael Lavista realiza la primera cirugía sobre una neoplasia intracerebral en 1891, y se publica en 1892. También se hace referencia, en esta serie informada por Lavista, al inicio de la cirugía de la epilepsia en México. El autor no ha encontrado, para el siglo XIX, otro informe de intervención sobre neoplasias intracerebrales en el ámbito hispánico.

History of cranial surgery, cerebral tumor surgery and epilepsy surgery in Mexico

\section{Summary}

The first rapport of intra-cerebral tumor surgery was provided by Bennett \& Goodle, in London, 1884. Worldwide this kind of surgery was performed in France by Chipault, in Italy by Durante, in the United States by Keen and in Deutchland by Krause \& Oppenheim. Lavista in Mexico City operated on intra-cerebral tumor in 1891, and the rapport was printed in 1892 . In the same publication, Lavista exhibited the first cases of epilepsy surgery. Since now, it is the first rapport of this kind of surgery in the Spanish spoken world.

\section{Introducción}

Los tumores intracraneanos en el adulto están en el $7^{\circ} \mathrm{u}$ $8^{\circ}$ lugar dentro de la patología neoplásica. En el niño se presenta como la segunda patología neoplásica más frecuente, encontrándose solo después de las hematopoyéticas, y

Recibido: 1-12-08. Aceptado: 25-02-09 como la primera patología neoplásica sólida ${ }^{22,69,70}$.

Presento en forma especial las contribuciones quirúrgicas hechas en este campo en México, por Rafael Lavista (figura 1) quien, en 1892, publicó una serie de cirugías cerebrales, con el caso de un tumor cerebral intraparenquimatoso, en un paciente pediátrico. Aureliano Urrutia (1904), Troconis (1911), Zuckermann (1935), Mario Fuentes y Manuel Guevara Oropeza (1939-1940) y Clemente Robles Castillo (1940 y 1944) publicaron sobre tumores craneanos y cerebrales ${ }^{17-19,31-33,52-56,64-66,72,73}$.

\section{Las fuentes}

Para la historia del abordaje quirúrgico de los tumores cerebrales en México se consultaron: el Periódico de la Academia de Medicina de Mégico (sic) (figura 2), México, desde 1836, que, sin solución de continuidad, se prolongaría con la Gaceta de la Academia Nacional de Medicina, que se publica hasta nuestros días. La revista de La Escuela

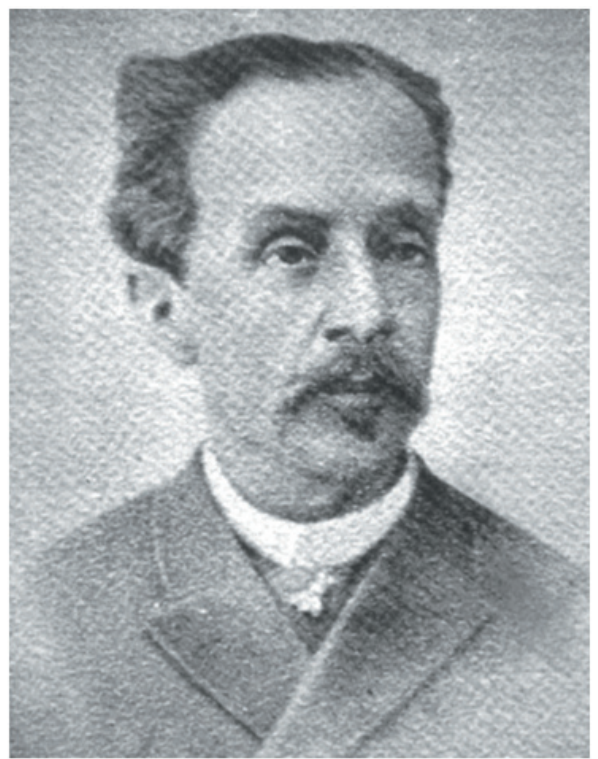

Figura 1. El doctor Rafaél Lavista 


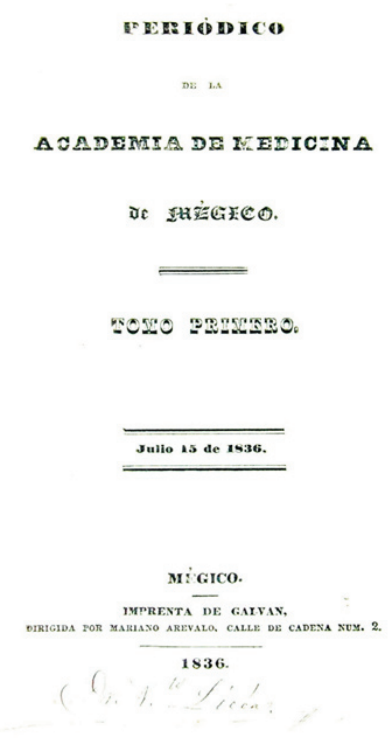

Figura 2. El Periodico de la Academia de Medicina de Mégico (sic), iniciada en 1836, fue el inicio de la actual Gaceta Médica de México, la publicación médica, actualmente activa, más antigua de América.

de Medicina, El Observador Médico órgano de la Sociedad Médica Pedro Escobedo, o la de El Porvenir, revista de los alumnos de la Escuela de Medicina de Santo Domingo y la Revista de Anatomía Patológica y Clínicas Médica y Quirúrgica ${ }^{14,15,23,40}$.

Especial importancia relevan las tesis para obtener el diploma de médico, de Bernardo Mortera y Franyuti así como la de Manuel Domínguez Galván, publicadas en 1892 y 1894 (figuras 3 y 4), respectivamente ${ }^{11,14,16}$.

También encontré ayuda en dos interesantes y buenas publicaciones de la primera mitad del siglo XX: la Revista Mexicana de Psiquiatría, Neurología y Medicina Legal y la otra son los Archivos de Neurología y Psiquiatría de México ${ }^{17-20,25,71-73}$. Hubo necesidad de recurrir a los trabajos de nuestros bibliógrafos e historiadores, que nos sirvieron en ocasiones de inspiración y en otras de firme referencia documental ${ }^{12,14,15,1,34-38}$.

\section{La cirugía de cráneo en México}

\section{Inicios}

La trepanación prehispánica en México y las trepanaciones con los métodos hipocrático-galénicos en el México del siglo XVI

En México hay evidencia de trepanaciones entre los aztecas, los mixtecas y los zapotecas, aunque en menor número que los cráneos europeos de la Prehistoria o los incas ${ }^{7,57,67}$.
EsCURLA Nacional DE MediciNa dE MEXICO.

ALGUUNAS CONSIDERACIONES

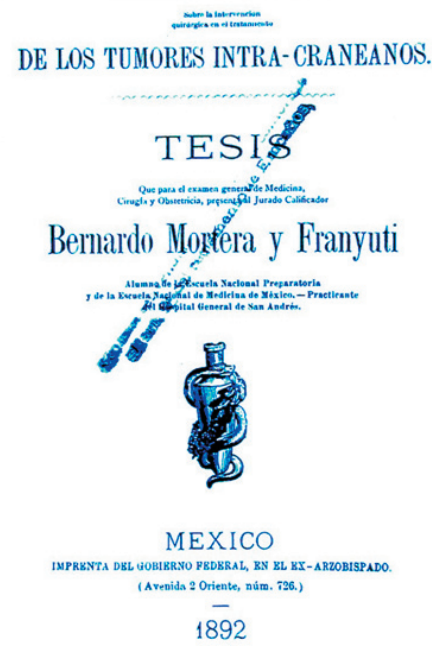

Figura 3. Tesis del doctor Bernardo Mortera y Franyuti, en donde se relata la cirugía de las epilepsias jacksonianas y la del tumor cerebral.

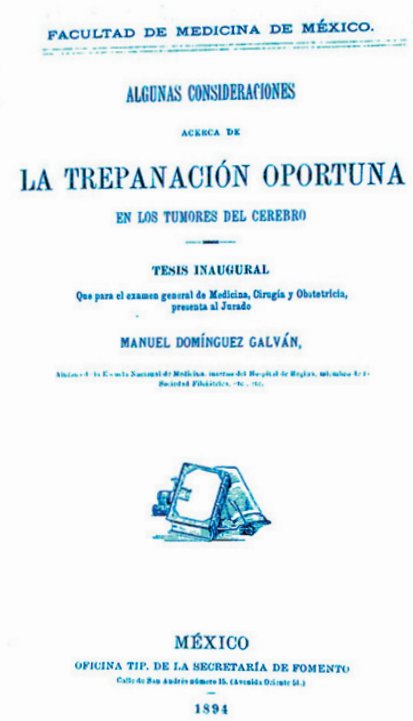

Figura 4. Tesis del doctor Manuel Domínguez Galván, en donde se presenta otra cirugía de cerebro, en el contexto de una epilepsia bravais-jacksoniana.

España trajo consigo el gran corpus médico de la Antigüedad Clásica y de la Edad Media, que estuvo constituido básicamente por los escritos de Hipócrates de Cos (480370 a. C.) y Galeno de Pérgamo, (130-200 d. C.) con las interpretaciones y comentarios de todos los autores que les sucedieron: Pablo de Egina, Avicena, Averroes, Abulcasis, Guy de Chauliac, los Saliceto, Juan de Vigo y muchos más ${ }^{3-}$ 8,13,21,24,34-39,41,57-62. 
En la ciudad de México, como herederos de la Antigüedad Greco-Latina, tres son los autores que mencionan estas técnicas. El primero es Pedro Arias de Benavides (15211570?), cirujano español originario de Toro, en Zamora, que ejerció en México, entre 1554 y 1564, y al regresar a España publicó en Valladolid, en 1567, en el libro Secretos de Chirurgía, el caso de un traumatismo craneano grave que requirió cirugía en 1561 en la ciudad de México, por una fractura hundida, conminuta, coma profundo y exposición de masa encefálica, todo con una evolución satisfactoria. El paciente fue un niño de 13 años apellidado Vergara ${ }^{1,4,6,34-}$ 37,58-62.

Después de Arias tenemos a Alonso López de Hinojosos, también español, nacido en Los Hinojosos (1525-1579), Provincia de Cuenca. Fue López de Hinojosos cirujano hábil que además tiene el mérito de haber publicado el primer libro de cirugía de América en 1578, salido de las prensas de Antonio Ricardo, piamontés; la famosa Summa y Recopilación de Chirurgia ${ }^{5-7,21,34-39,58-62}$. López describe, cuidadosamente, los métodos galénicos de trepanación, con sus respectivas indicaciones.

El más cultivado de los médicos que publicaron al respecto en México fue el padre Agustín Farfán (1532-1604), originario de Sevilla, España. Su primera publicación llamada Tractado de Anothomia y Chirugia, también impresa por Ricardo, data de 1579, un año después de la de López. Dentro de los aspectos anatómicos encontramos la primera descripción detallada de una anatomía del cerebro, de corte meramente galénico, y sus técnicas para la trepanación y el tratamiento de las fracturas de cráneo son también galénicas, como las de Arias y López $z^{4,6,7,34-39,58-62}$.

Las publicaciones del siglo XVII y XVIII son también de calidad, sobre todo la enciclopedia médica de Juan de Barrios, publicada en el siglo XVII. En el siglo XVIII se inaugura en México y a nivel continental la publicación de revistas médicas, con el Mercurio Volante, de José I. Bartolache, aunque sin referencias a cirugía de cráneo ${ }^{1,3-9,13,21,23,34-}$ 40,47,59-62.

El siglo XIX se inició todavía con la Real y Pontificia Universidad de México y sus programas de enseñanza medievales. No sería sino hasta 1833 con Casimiro Liceaga y Valentín Gómez Farías, en el Establecimiento de Ciencias Médicas, que se modernizarían los planes de estudio de la medicina en México, con la consecuente modernización de las ideas acerca de la cirugía de cráneo ${ }^{8,9,16,34,36}$.

\section{Cirugía del traumatismo craneano en el México del siglo XIX y principios del $X X$}

José María Terán y Luis Hidalgo y Carpio publicaron, en 1842, un caso de traumatismo craneano, en el Periódico de la Academia de Medicina de Mégico (sic) ${ }^{63}$. Pomposo Hinojosa informó (1856-1858) de una "Herida de la cabeza curada por la operación del trépano"28. Hidalgo y Carpio, en 1864, publicó una serie de pacientes operados de cráneo por traumatismo. En este tiempo también describe, además de la duramadre y la piamadre, a la aracnoides. Una mortalidad del $50 \%$ es informada por Hidalgo y Carpio, únicamente para la patología neuroquirúrgica traumática ${ }^{26,27,29}$. En 1870, Francisco Montes de Oca presentó un trabajo sobre una "Herida penetrante del cráneo. Extracción del cuerpo extraño. Accidentes consecutivos. Muerte del enfermo. Autopsia”, esto en la Gaceta Médica de México, en el tomo 54. En 1879, Ricardo Egea presentó un trabajo sobre una "Herida de cabeza en la región frontal; fractura y hundimiento del hueso; trepanación sobre el seno longitudinal superior; curación", en la misma Gaceta, en el tomo $14^{12}$. Para el año de 1885 , en el tomo 20 Francisco Marín informó sobre una "Herida de la cabeza por arma de fuego, Trepanación. Curación" ${ }^{43}$. En la revista La Escuela de Medicina, de Garay, analizó en 1891 una corta serie de 4 casos de traumatología cráneo-encefálica ${ }^{20}$. En la misma revista Pagenstecher (1899) comunicó sus "Reflexiones sobre la trepanación y sus indicaciones en las afecciones traumáticas". Esta es una pequeña serie de cinco $\operatorname{casos}^{50}$. La utilización de la trepanación en repetidas ocasiones, en un mismo paciente y por la misma causa, es manifestada por Tobías Núñez en el Periódico de la Academia de Medicina de México, en $1908^{48}$.

José Ramón Icaza publicó en 1910 “Un caso de epilepsia jacksoniana curado por la trepanación”, en la Gaceta Médica de México, en una operación de cráneo, por una fractura hundida que ocasionaba crisis epilépticas bravaisjacksonianas $^{30}$.

\section{Inicios de la cirugía de tumores intracraneanos y de la epilepsia en México, siglo XIX}

Es en el siglo XIX, fecundo en personalidades médicas de primera línea, cuando se inicia este tipo de cirugía en México ${ }^{8,16}$.

La primera referencia encontrada por mí, publicada en México, de un tumor cerebral, es la de Fernando Zárraga en la revista de La Escuela de Medicina, en 1891, "Un caso de tumor cerebral”, esto en 1891. El caso es de necropsia ${ }^{71}$.

La tesis para obtener el título de médico de Bernardo Mortera y Franyuti, en colaboración con José Uribe y Troncoso, fechada de 1892, trata sobre Algunas consideraciones sobre la intervención quirúrgica en el tratamiento de los tumores intracraneanos, México $1892^{46}$.

En ella se hace referencia a cinco casos operados por Rafael Lavista de lesiones cerebrales, dos quísticas en 1890, un tumor intracerebral en 1891 y además dos traumatismos craneanos, los cinco casos con una epilepsia bravais-jackosianana. Las lesiones quísticas correspondieron al mismo paciente, quien presentaba un quiste hidatídico. Este caso 

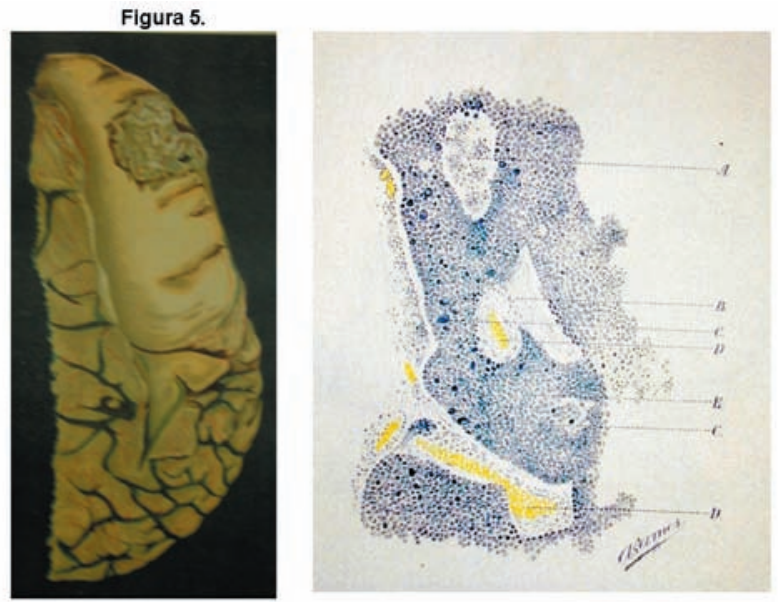

Figura 5. Primer informe gráfico en México de un tumor intracerebral, con examen macroscópico y microscópico, ambos en litografía, publicados por el dpctor Lavista en su Revista de Anatomía Patológica y Clínicas Médica y Quirúrgica, en 1899

se presentó en el Congreso de Moscú, de 1890, en la comunicación del Dr. Lavista: Contribution à l'étude des localisations cérébrales au point de vue de la Clinique. Juillet $1890^{32}$. Esta tesis se presenta como el estudio más completo publicado en México, para la época, sobre este tipo de epilepsia y se constituye, además, en el primer informe de un tumor intracerebral operado con éxito y los primeros informes en México sobre cirugía de la epilepsia. Hasta donde las investigaciones del autor han podido llegar, también lo son para el resto del mundo hispano-parlante ${ }^{42,68}$.

En el primer capítulo de la tesis de Mortera se menciona la importancia de las zonas de localización, que permitirán abordar seguramente esta patología y las contrapone con las llamadas zonas latentes, que no dan una buena localización de la neoplasia cerebral. Para esto propone dos métodos, basados en la localización del bregma y la situación que éste guarda con relación a la emergencia del surco central sobre el borde dorsal del hemisferio; de 4.5 a $5.3 \mathrm{~cm}$ por atrás del bregma:

1. Escuadra flexible de Broca: con la cual era posible localizar la línea aurículo bregmática sin mayor problema (Figura 6).

2. El método más sencillo de Lucas Championniere, que se valía de un simple cartón para localizar la mencionada línea (Figura 7).

El interesante segundo capítulo trata sobre las localizaciones cerebrales. Hace un recapitulativo histórico sobre el tema, que es realmente interesante puesto que lo inicia con una crítica a las afirmaciones galénicas, aun esgrimidas por algunos médicos de la época, de que los giros y la corteza cerebral en general “... podía ser cortada, destruida o mal-
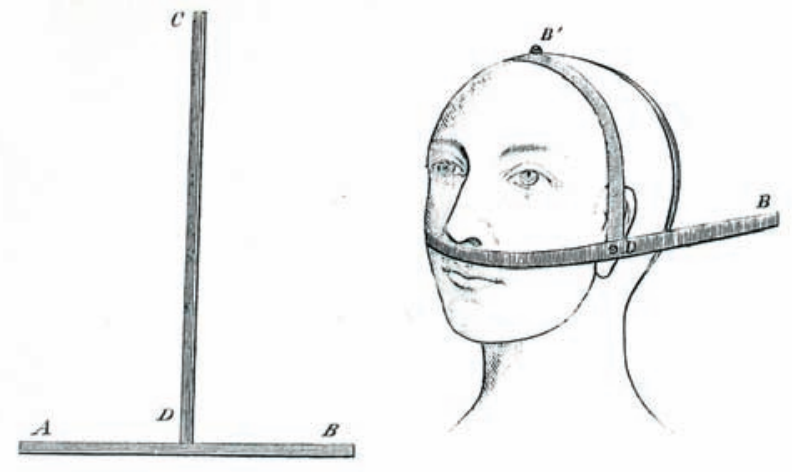

Figura 6. Escuadra flexible de Broca.

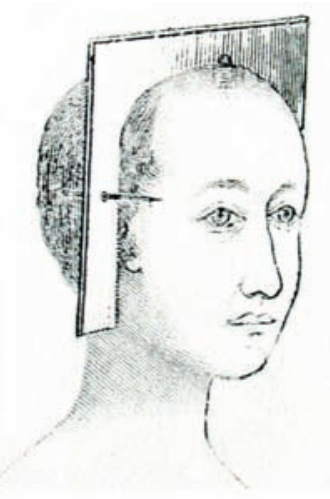

Figura 7. Escuadra de Championiere.

tratada de cualquier otro modo en los animales sin provocar fenómenos de sensibilidad o movimiento...". Nombra a los principales investigadores europeos dentro de este campo: Gall, Broca, Charcot y Jackson, entre otros.

El capítulo tres trata de la epilepsia jacksoniana. Este tipo de convulsiones habían sido ya descritas antes, desde los lejanos tiempos de Hipócrates y Galeno. Menciona, con justicia, que la primera descripción cuidadosa sobre este tipo de epilepsia es de Bravais, en 1827, y dice que no encontró eco a causa del atraso, en esa época, en los estudios anatomo-patológicos.

En cuanto a la producción de la convulsión, invoca fenómenos eléctricos y cita a Jackson, así como a Todd y Robertson, comparando a las neuronas, "una celdilla cerebral", con una botella de Leyden. La parálisis post crítica traduciría el agotamiento de las reservas eléctricas de esta celdilla.

La división de las crisis que da es la de Bravais, no la de Jackson, anotando los autores que se han ocupado particularmente de cada una de éstas:

1. Tipo facial. Demongeot de Confevron, Hitzig, Wernher, Vermeuil, Gowers, Charcot y Bell. Crisis faciales y de 


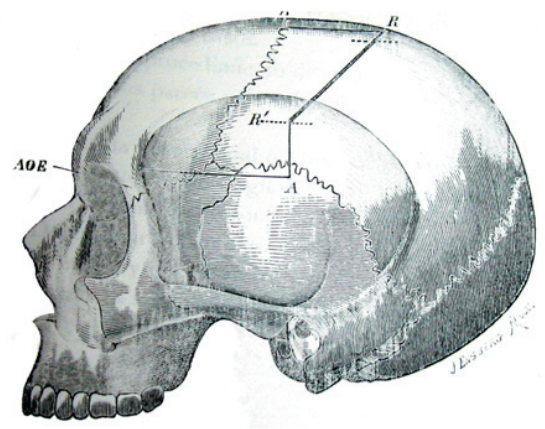

Figura 8. Craniometría con el método de Broca.

la musculatura del cuello. Desviación de comisura y lengua hacia fuera, contractura del esternocleidomastoideos contralateral y desviación de los ojos hacia arriba.

2. Tipo braquial. Jackson, Charcot, Glicky, Mahot, Bernhardt, Henrot. Se inicia en los dedos de la mano, subiendo en nivel e intensidad, llegando hasta el hombro del mismo lado, con frecuente participación de cara y cuello.

3. Tipo crural. Jackson y Griesingier.

\section{Las cirugías, sobre lesiones cerebrales y para tratar la epilepsia}

\section{Primer caso, 1890.}

Cirugía sobre lesiones quísticas: es el caso de un hombre de 25 años, que fue sometido a 2 intervenciones, siendo la primera por un quiste hidatídico y la segunda, al parecer, por un hematoma subdural crónico, con membranas. Esta patología no es precisamente tumoral, pero los razonamientos y las técnicas empleadas por Lavista, son los de una cirugía tumoral.

Segundo caso, primera cirugía por tumor intracerebral en 1891.

El tumor cerebral fue catalogado por Lavista como "gliosarcoma" cerebral, muy posiblemente una metástasis del tumor de partes blandas escapular derecho. Dadas las competencias del cirujano dentro del campo de la anatomía patológica, es casi seguro que el tumor se haya sometido a un análisis microscópico.

Se trató de un niño de 15 años, natural de Celaya, Guanajuato, que ingresó en el Hospital el primero de abril de 1891. Los antecedentes fueron un padre epiléptico y un hermano fallecido por meningitis y otro por "congestión cerebral". Dos años antes inició con la presencia de tumor escapular derecho. Fue intervenido en cuatro ocasiones, siendo la primera una punción, recidivando después de cada exéresis, e ingresando en el Hospital para la cicatrización de una gran pérdida de substancia en la piel escapular

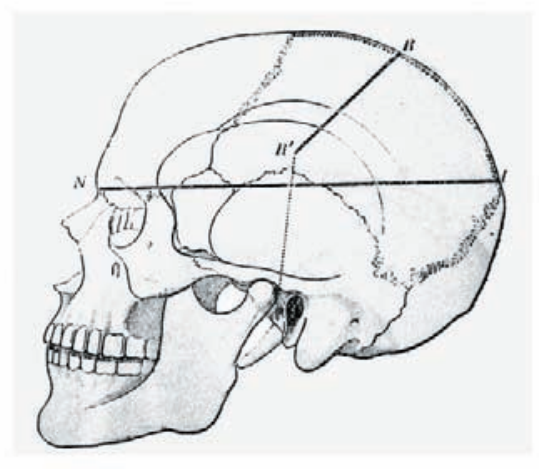

Figura 9. Craniometría con el método de Championiere.

derecha, por las repetidas operaciones.

Hacia el día 10 de abril, en el transcurso de la cura y dentro de un contexto caquéctico, presentó monoparesia inferior izquierda, que avanzó hasta no poder sostenerlo, posteriormente hemiparesia, con crisis convulsivas hemicorporales izquierdas, con generalización posterior y hemiplejia derecha, estado de coma profundo y midriasis paralítica derecha. Había también una anemia consignada a $50 \%$ de la hemoglobina y una hipertermia de $40^{\circ} \mathrm{C}$.

El diagnóstico presuncional contempló una lesión cerebral que podría ser de varios tipos: infección con absceso, hemorragia, hematoma y por supuesto una lesión neoplásica, que fue la opción retenida. Se preparó al paciente con el empleo de antiflogísticos, hielo sobre la cabeza, un purgante drástico y evacuación de la vejiga mediante cateterismo.

Se decidió realizar una craneotomía, el día 13 de abril, con el objetivo de extirpar el tumor y mejorar la epilepsia. Se realiza una incisión sobre la región central, en su parte posterior, de $14 \mathrm{~cm}$ de largo. Se aplicó sobre el hueso una trefina inglesa, sacando una rondela de hueso de cinco $\mathrm{cm}$ de diámetro, a dos cm de la sutura sagital. Se presentó, al retirar el hueso, una herniación del cerebro, aún con la dura madre íntegra. Esta última se cortó en cruz, encontrándose las circunvoluciones deformadas, haciendo pensar en la existencia de una masa o una colección por debajo. Se puncionó obteniendo un poco de sangre. Posteriormente se logró hacer salir por el agujero de la punción una cierta cantidad de "(...) pus y material caseoso(...)". Se lavó y esterilizó la cavidad con agua a $40^{\circ} \mathrm{C}$. Se realizó inspección digital con el dedo índice de la mano izquierda, que se hundió en toda su longitud. Se pudo apreciar por esta palpación una estructura de paredes lisas, pulidas y como substancia cerebral condensada. La lesión era de forma ovoide, gruesa y voluminosa. Se practicó una exéresis digital, con los dedos índice y medio izquierdos del doctor Rafael Lavista. Una ablación completa de la lesión fue posible, sin pérdida de sangre. Después de lavado de la 
cavidad y canalización al exterior, se cerró por planos.

Los resultados postoperatorios tempranos fueron excepcionalmente buenos, con el despertar tranquilo del enfermo, la regresión de la hemiplejia izquierda, la desaparición de las crisis convulsivas y la normalización del diámetro pupilar derecho y sus reflejos. La temperatura bajó hasta $37.5^{\circ} \mathrm{C}$.

El Paciente falleció el día primero de julio del mismo año, sobreviviendo a la operación poco más de un mes y medio, sin datos de infección quirúrgica pero, a decir de los autores, por la progresión de su problema neoplásico, que ocasionó hernia cerebral por la región de la craneotomía, que era evidente en el curso de las curaciones.

Otra tesis sobre la cirugía de tumores cerebrales es la de Manuel Domínguez Galván, fechada de 1894 y que se intitula Algunas consideraciones de la trepanación oportuna de los tumores del cerebro, México $1894^{11}$. En esta se informa de un caso de un quiste cerebral operado también por Lavista, en un joven de 22 años.

Aparte de las primicias en cuanto a la cirugía de la epilepsia y de un tumor intracerebral, en el ámbito hispánico, contamos con la cuidadosa descripción de su técnica de disección digital, que se da 40 años antes de que Dandy hiciera su publicación al respecto, en $1932^{10}$.

A Aureliano Urrutia corresponde el honor de haber publicado una segunda corta serie de tres lesiones cerebrales operadas en la ciudad de México, de las cuales una también corresponde a un tumor. La publicación es de 1904, en el Periódico de la Academia Nacional de Medicina de México ${ }^{65}$. Urrutia dice que las referencias empleadas para localizar el surco de Rolando por los cirujanos galos antes mencionados, no correspondían de manera completa a la morfología cráneo-cerebral del mexicano, y nos recomienda un método muy complicado, en donde es necesario realizar, además de las líneas y medidas dadas por los franceses, una serie de complejas operaciones matemáticas, con las cuales se puede definir la zona cerebral central del mexicano. Estas elucubraciones yo las considero ociosas e inútiles.

Quizás lo más interesante del artículo es que nos refiere 3 casos de lesión cerebral operados, de los cuales uno es un niño de 13 años, con el diagnóstico de "gliosarcoma" difuso, el único que presentó un tumor cerebral. Es este, entonces, el segundo caso publicado de una patología tumoral intracerebral operada en México, y como el de Lavista, es también un caso pediátrico. Otras dos operaciones cerebrales se realizaron sobre "quistes".

El paso Aureliano Urrutia por la Dirección de la Escuela de Medicina es controvertido, puesto que según Francisco Fernández del Castillo, Urrutia impidió que la mencionada Academia sesionara en el Palacio de Santo Domingo, sede de la Escuela de Medicina, lo que es tomado como una injuria severa por parte del historiador. Se le acusa también de haber depauperado la biblioteca de la mencionada Escuela, al tirar a la calle algunos ejemplares de la apreciada Revue de Physyologie de Francia ${ }^{14}$.

Troconis Alcalá informó en la Gaceta Médica de México, fechado el 26 de julio del 1911, de un tumor de cráneo, no cerebral, operado en México en el Hospital General. Se trata del caso de un niño, del sexo masculino, de 8 años, quien se presentó con una tumefacción en la región temporal izquierda, originándose en el músculo temporal, deformando la región temporal y la cara del lado izquierdo. La extirpación fue total y a pesar de esto hubo una recidiva que fue tratada con radiaciones, "rayos ultraviolados" (sic), nos dice el Dr. Troconis, que no mejoraron al paciente, que fallecería 4 meses después de la cirugía. El resultado del estudio anatomopatológico fue el de un sarcoma quístico. Es importante repetir que es uno de los primeros informes mundiales de un tumor de cráneo tratado con radiaciones ${ }^{64}$.

He encontrado un lapso prolongado, desde 1911 hasta 1935, en donde no he podido localizar publicaciones correspondientes a la cirugía de tumores de cráneo y cerebrales. Si bien es cierto mi búsqueda no ha sido exhaustiva, creo también que ha sido, hasta el momento, lo más completa posible. Es de pensar que las convulsiones sociales que sacudieron a México en esos años no permitieron que se publicara mucho sobre la cirugía de tumores cráneocerebrales, que seguramente sí debió haber existido.

Conrado Zuckerman publica en 1935 una pequeña monografía sobre el "Concepto Anatomo-Patológico de los Tumores Cerebrales", en la Revista Mexicana de Psiquiatría, Neurología y Medicina Legal, seis años después de la monografía de Bayley y Cushing sobre los gliomas cerebrales y apenas 2 años después de las publicaciones independientes de Bayley y Love sobre el tema. Las inquietudes de Zuckermann abarcaban también los métodos diagnósticos que facilitarían el ejercicio de la cirugía neurológica. En 1937 nos encontramos un artículo muy completo, publicado por él en la misma revista, que trataba de la "Técnica y Resultados de la Aeroencefalografía por vía lumbar". Nos puntualiza que este estudio debe de ser limitado para los tumores intracraneanos supratentoriales e informó sobre 3 casos de tumor cerebral en donde este estudio fue de gran utilidad. Mencionó y comentó el resto de indicaciones para la realización del estudio ${ }^{72,73}$.

En 1938 el doctor Manuel Guevara Oropeza, psiquiatra, publicó el caso de un tumor del ángulo pontocerebeloso, en la revista Archivos de Neurología y Psiquiatría de México $\mathrm{y}$ el diagnostico presuncional de neurinoma del VIII nervio izquierdo ${ }^{25}$. El neurocirujano fue el doctor Mariano Vázquez y no se trató de un tumor, sino de un cisticerco, que había anidado sobre el VIII nervio izquierdo, en el ángulo pontocerebeloso. Aparte de un abordaje clínico de primera calidad, hecho por Guevara, los estudios de 
gabinete fueron: radiografías simples de cráneo, con impresiones digitiformes, borramiento de clinoides posteriores y ensanchamiento del conducto auditivo interno izquierdo. La ventriculografía occipital bilateral, hecha por Vázquez, demostró la existencia de una hidrocefalia. La paciente falleció dos días después de la cirugía. El doctor Isaac Costero realizó la necropsia, que evidenció un quiste de cisticerco interpeduncular, un abseso pequeño y una aracnoiditis crónica, además de la ya mencionada hidrocefalia.

En el mismo año y en la misma Revista, el doctor Mario Fuentes, también psiquiatra, publicó una "Nota Clínica sobre un caso de meduloblastoma con síndrome adiposo genital", en un niño de 13 años. El cirujano fue el doctor Mariano Vázquez, y en este escrito se aclara que la cirugía se llevo a cabo en el Hospital General. El caso es interesante puesto que el meduloblastoma del IV ventrículo invadía el III, pasando por el acueducto de Silvio. Se le realizó un excelente estudio clínico, con el diagnóstico de síndrome de Babinski-Frölich. Los estudios de imagen mostraron una hipertensión endocraneana crónica, que la clínica asentaba con una atrofia papilar bilateral. Durante la operación no se pudo abordar al tumor. El diagnóstico definitivo fue de autopsia ${ }^{17}$.

En el mismo año de 1938 Fernando Valdés Villareal operó, con éxito, el caso de una osteítis fibrosa temporal izquierda intracraneana, en una mujer de 56 años que se presentaba con una sordera vieja bilateral, crisis convulsivas generalizadas, cefaleas, trastornos de la memoria, bradipsiquia y aumento de los reflejos osteotendinosos. La publicación la realizó Samuel Ramírez Moreno en la Revista Mexicana de Psiquiatría, Neurología y Medicina Legal ${ }^{52}$.

Conrado Zuckermann operó en estos tiempos un caso de quiste hemorrágico del hemisferio cerebeloso izquierdo, en una mujer de 38 años, que se presentó con crisis convulsivas parciales y posteriormente generalizadas, además de cefaleas occipitales y posteriormente monoparesia inferior izquierda. Al examen hay un síndrome cerebeloso, estático y dinámico, bilateral predominando a la izquierda y edema de papila. No hay comprobación patológica de tumor. Esta publicación la realizaron los estudiantes de medicina Manuel Velasco y Juan A. Sanen, en la misma Revista Mexicana de Psiquiatría, tampoco tenemos referencias bibliográficas ${ }^{66}$.

El doctor Mario Fuentes tomó, seguramente, el gusto por las publicaciones neuroquirúrgicas, puesto que en 1939 y 1940 hace otras dos publicaciones, sobre tumores cerebrales, en los Archivos de Neurología y Psiquiatría de México.

El artículo del 1939 lo publica teniendo como coautor al neurocirujano, doctor Mariano Vázquez, con el título de "Un caso de tumor del tercer ventrículo". Aunque no se refiere ni la edad ni el sexo, inferimos por la lectura del artículo que se trata de un niño, del sexo masculino. De nuevo encontramos un fino análisis clínico del doctor Fuentes, que instaura el diagnóstico de una hipertensión endocraneana crónica. Las radiografías de cráneo y las ventriculografías diagnosticaron una hidrocefalia. La intervención, por medio de una callosotomía, permitió la extirpación de un "cistoma hipofisiario", de 40 gramos de peso. La anatomía patológica corrió a cargo del doctor Isaac Costero. De nuevo, a pesar de una buena cirugía, el paciente falleció unos días después ${ }^{18}$.

El trabajo del 1940, que publica únicamente bajo su nombre, se intitula: "Tumor del tercer ventrículo. $\left(3^{\mathrm{er}}\right.$. Caso)". Vemos bien que ya era tomado a justo orgullo el haber podido publicar una serie tan grande de estas neoplasias, a pesar de no haber sido el cirujano. Este tercer caso es también un paciente pediátrico, de 16 años, del sexo masculino, y como en el primer caso, éste presenta un síndrome de Babinski-Frölich. Aquí también vemos un fino análisis clínico, que determina una hipertensión endocraneana crónica además de trastornos del carácter. Las radiografías de cráneo confirmaron esta hipertensión y la ventriculografía aérea puso de manifiesto una exclusión del III ventrículo, que hizo sospechar la presencia de un tumor en esta región. No menciona el nombre del doctor Mariano Vázquez como el neurocirujano que realizó la ventriculografía, pero es de pensarse que fuera él. El mal estado del paciente no permitió la exéresis tumoral y el diagnóstico lo dio la necropsia y el estudio anatomopatológico, tratándose de un gran tumor del III ventrículo, con una gran porción infundibular y que llegaba hasta los límites del cuerpo calloso y la parte posterior del III ventrículo. El estudio histopatológico, también del doctor Isaac Costero, especificó que se trataba de un "glioepitelioma", (ependimoma). Fuentes fue citado abundantemente por Robles, quien discute la pertenencia al III ventrículo tanto del tumor hipofisiario como del meduloblastoma ${ }^{17-19,54}$.

Zuckermann publicó en 1939, en la misma revista, una monografía sobre tumores cerebrales. Menciona a Manuel Balado y Ramón Carrillo y sus aportaciones al diagnóstico imagenológico con su método de iodoventriculografía e iodoventriculoscopia, hechas con lipiodol inyectado en los ventrículos laterales.

En este escrito de nuevo se nos confirma que los cirujanos de cráneo y cerebro en México preferían la anestesia local a la general, como también la prefería Thiery de Martel $^{44,68,73}$. Desgraciadamente Zuckermann no ilustra con casos clínicos este escrito.

La bibliografía más amplia sobre el tema, la exhibe Zuckermann en esta muy correcta monografía. Las citas son a: Alfred Adson, Percival Bailey, De Viggo Christiansen, Harvey Cushing, Percival Bailey, Louise Eisenhardt, Walter Dandy, Lewis Dean, Leo Davidoff, 
Loyal Davis, Cornelius Dyke, Klipel, Winchell McKraig, James Kernohan, Lipscomb, Shelden, Egas Moniz, Clement Masson, Jean Paillas, Georges Guillain, Neuberder, Paulian, Roussy, Lhermitte, Cornill, Ody, Cobb, Pilcher, Hug Wilson, Puusepp, Ernest Sachs, Israel Strauss, Max Thorek, Cecil Wakeley, Ramón Carrillo, Manuel Balado, Alberto Gutiérrez, Julio Hanon, Francisco Convers, Teodoro Flores Covarrubias, Samuel Ramírez Moreno, Luis Sánchez Bulnes, Severino Vieira, Miguel Lavalle, Alberto Fernández Salarraguí, Manuel Guevara Oropeza, José Rojo de la Vega, Mariano Vazquez, Manuel Velasco y Juan Sannen ${ }^{73}$.

El patólogo español Isaac Costero y H. Berdet, publican en 1939 una serie de 135 tumores hipofisiarios, en las Monografias de la Sociedad Médica del Hospital General de México ${ }^{55}$.

\section{Época contemporánea; inicio de las grandes series}

En 1940 se presenta publicada una serie neuroquirúrgica de tumores intracraneanos, en la Gaceta Médica de México, todos ellos operados por Clemente Robles Castillo, en el Servicio de Cirugía Nerviosa del Hospital General de México. Siete fueron los casos que presentó Robles, todos ellos perfectamente descritos y documentados. Este fue su trabajo de ingreso a la Academia Nacional de Medicina ${ }^{53}$.

Los casos, por orden de edad fueron:

Un niño de 9 años, operado de un epitelioma hipofisiario; un hombre de 35 años, de un adenoma cromófobo de hipófisis; un hombre de 36 años de un tumor calcificado frontopolar derecho; una mujer de 40 años, de una hoz calcificada; una mujer de 46 años, de un quiste dermoide parietooccipital izquierdo; un hombre de 48 años, de un glioblastoma multiforme y una mujer de 50 años, con un adenoma cromofobo de hipófisis.

Robles recomendaba un equipo multidisciplinario, bien organizado, para poder abordar este tipo de tumores. No deja de manifestar reconocimiento a Isaac Costero patólogo, Magín Puig Solanes oftalmólogo, Roberto Gamboa Acosta neurólogo y Carlos Coqui radiólogo. Agradece también a Ignacio Chávez por su apoyo.

La bibliografía en esta serie es interesante, ya que se apoya en las piedras angulares para la cirugía de tumores intracraneanos de aquel entonces: Cushing, Clovis Vincent, David, Puech, Heuer, Wilder Penfield, Leo Davidoff, Dike, Jonson, Costero y Gregorio Marañón ${ }^{53}$.

Gonzalo Castañeda en la réplica al trabajo de Robles, hizo referencia a "la trepanación" efectuada por Lavista en el último decenio del siglo XIX, sin hacer mención de la cirugía cerebral realizada por él.

Héctor Romo Bolán informó de un caso en 1941 en la Revista Mexicana de Psiquiatría, Neurología y Medicina
Legal, desde Acámbaro Guanajuato, sobre un "Meningeoblastoma terebrante izquierdo" de una niña de 16 años, ya casada y con un hijo, que llegó con él, ginecoobstetra, para consulta ginecológica por trastornos de la menstruación. Romo realizó la exéresis completa de la lesión ${ }^{56}$.

Robles Castillo en el volumen de 1942 de la Gaceta Médica de México hace la primera publicación, en México, de un quiste coloide del III ventrículo ${ }^{54}$. Se trató de un hombre joven de 22 años, con el cuadro clásico de cefaleas agravadas con cambios de posición de la cabeza, sobre todo si éstos eran bruscos.

Discute ampliamente sobre la cirugía del III ventrículo en el México de la época. Menciona a Fuentes y sus publicaciones, con 3 casos de tumor del III ventrículo. Robles nos dice que en su servicio de Neurocirugía del Hospital General tienen 5 casos, uno de ellos compartido con los casos publicados por Fuentes, lo que hace un total de 7 tumores localizados en el III ventrículo: 1 meduloblastoma, 1 cistoma hipofisiario, 1 ependimoma, 1 quiste coloide, 1 carcinoma hipofisiario, 1 adamantimoma hipofisiario y 1 tuberculoma.

La bibliografía es selecta: D. Andersón, F. Andersón, Walter Dandy, Fuentes, Fulton, Gardner, Jaeger, Jefferson, Larson, Nieven, Neustaedter, Patterson, Shaver, Stookey. Traut, Trescher y, Zeitlin.

En 1942, Jorge G. Pavón Abreu publicó en la Revista de Psiquiatría, Neurología y Medicina Legal, dos casos de tumor del ángulo ponto-cerebeloso ${ }^{51}$.

Correspondería a Clemente Robles Castillo el publicar otra serie de neoplasias intracraneanas en 1944, dentro de la Gaceta Médica de México y que hace referencia a operaciones realizadas entre 1938 y 1944. Los casos fueron del Hospital General de México Eduardo Liceaga, algunos de la práctica privada y otros más del Hospital Infantil de México Federico Gómez.

Consta esta serie de 100 casos, pero 25 fueron cisticercos, 11 tuberculomas, dos osteomas de la hoz del cerebro, un quiste dermoide y un goma sifilítico. El total de tumores operados sería entonces de 60 , que son ya bastantes $^{55}$.

Los más frecuentes fueron los meningiomas, seguidos de los astrocitomas, los glioblastomas, los adenomas cromofobos de hipófisis, los craneofaringiomas y otros.

Tenemos entonces que los más frecuentes fueron los gliomas en general, con 20 casos, representando un 30\% del total, seguidos por los adenomas hipofisiarios y los meningiomas, con 11 casos cada uno. Los craneofaringiomas fueron 7; el total de tumores de la región hipofisiaria fue de 21 casos, es decir un poco más de $30 \%$. Los neurinomas fueron 3. Como podemos ver, el resto de los tumores tuvo un solo caso.

Muy interesante es revisar los resultados que nos dan la 
idea de lo difícil del inicio de esta cirugía en México:

Meningiomas, 11 casos: 5 casos de mortalidad operatoria, 5 curados y uno con hemiplejia.

Adenomas hipofisiarios, 11 casos: 1 un solo caso de mortalidad operatoria y otro deceso por recidiva, de un paciente que no quiso ser reoperado.

Craneofaringiomas, 7 casos: 5 casos de mortalidad operatoria, 2 pacientes vivos, de los cuales uno ciego, que ya lo estaba antes de la cirugía.

Gliomas, 10 casos; ya se ha discutido.

Aclarando que apenas la primera serie de 60 casos de esta patología informada en México, nos hace saber sobre sus dudas y sus primeros pasos, “... a menudo inciertos y vacilantes...”. Afirma que es uno de los capítulos más difíciles de toda la cirugía. Su opinión toma relieve, puesto que él hacia cirugía general y fue además fundador del servicio de cirugía cardiovascular del Instituto Nacional de Cardiología Ignacio Chávez.

Nos da una mortalidad total de $38 \%$. Hace las pertinentes observaciones sobre la predominancia de problemas tales como la cisticercosis y tuberculosis, que son una muy pequeña parte de lo publicado en Europa y los EUA. Se queja de que las lesiones que llegan son muy grandes y que esto empobrece el pronóstico.

Sus deseos son alcanzar las tan agradables cifras de una mortalidad operatoria máxima de $10 \%$, como lo afirmaba en su tiempo Cushing.

También se hace una pregunta, que más tarde él mismo se respondería; “... ¿Vale la pena operar la cisticercosis?...". Sus inquietudes irían hasta la experimentación y el inicio del control farmacológico de la cisticercosis, 40 años después de haberse preguntado él mismo esto.

La bibliografía de este último articulo nos muestra la biblioteca del Dr. Robles: Bayley, Costero, Cushing, Dandy, Guevara Oropeza, Ochoterena y otros.

Es hasta esta última publicación del Dr. Robles que avanzaré esta investigación.

\section{El inicio de la neuropatología en México}

El mérito de iniciar las publicaciones de tipo meramente neuropatológico, en México, corresponde a Rafael Lavista, quien en 1896 funda la Revista de Anatomía Patológica. En un trabajo de 1899 , en referencia al sistema nervioso nos enteramos de que existían 150 estudios microscópicos y algunas piezas del sistema nervioso central con tumores del cerebro, del cerebelo y parasitosis cerebrales, entre otros. En este mismo volumen se exhibe una ilustración de un corte de hemisferio izquierdo, con una tumoración a nivel del segundo giro frontal. Hay otra ilustración, de una preparación microscópica de dicha lesión. Ambas son bellas litografías y las primeras imágenes publicadas de este tipo en México (Figura 5) 33 .
Neuroanatomía, neuroimagenología y neuroanestesia. Sus inicios en México

Hidalgo y Carpio describe en 1864, además de la duramadre y la piamadre, a la aracnoides ${ }^{26}$. Lavista utiliza métodos de localización cerebral, de Broca y Championiere, en su presentación para el congreso de Moscú en 1891, y en las tesis de Mortera y Domínguez ${ }^{32,46}$.

Los primeros informes en México sobre la aeroencefalografía lumbar son de Zuckermann, en 1937. Valdés Villarreal y Zuckermann (1938), Fuentes y Vázquez (1938-1940) y los siete primeros casos de Robles (1940), se acompañaron de RX simples de cráneo. Dos de los casos Fuentes, tres de los de Robles y en el caso de Zuckerman, se realizó una ventriculografía. En la serie del 1944 de Robles, las ventriculografías fueron $50 \%$ del total de casos, aproximadamente. Hasta el momento, no hemos encontrado referencias a arteriografías realizadas en México antes del $1944^{15,53-55,72,73}$.

La anestesia general iba progresando al ritmo de la cirugía, sin embargo, en México en los años 30 se operaba con anestesia local al cráneo y al cerebro ${ }^{44,52,68}$.

\section{Tratamientos complementarios. Inicio de la radioterapia en México}

Krause en 1909 publicó los primeros casos de irradiación de tumores hipofisiarios. Para un sarcoma del músculo temporal, operado en México en 1911, ya se estaba utilizando la radioterapia complementaria, tres años después de Krause. Robles (1944) prefiere la irradiación de adenomas eosinófilos, en lugar de una cirugía de exéresis total.

\section{Conclusión}

Como conclusión, considero necesario decir que los hombres que no conocen ni juzgan sus antecedentes, están condenados a que personas ajenas lo hagan por ellos. Del extranjero rara vez es posible esperar justicia. La casi totalidad de opiniones sobre la historia de la medicina mexicana, no hecha por mexicanos y en ocasiones hecha por mexicanos que emulan o copian una falsa o inexacta historia, nos la muestran pobre, parca y mezquina.

\section{Agradecimiento}

Los artículos de las revistas de Psiquiatría y Neurología, de los años treintas, me fueron proporcionados por la doctora María Cristina Sacristán.

\section{Bibliografía}

Las ilustraciones de la pieza anatomopatológica, macros- 
cópica y microscópica, están tomadas de la Revista de Anatomía y Patología Clínicas. 1899; tomo IV(9-10).

Las figuras de las craniometrías, así como de los instrumentos utilizados para ellas, se tomaron de la tesis de Bernardo Mortera y Franyuti.

1. Arias de Benavides, P.: Secretos de Chirurgia. Valladolid, impreso por Fernández de Córdova 1567. Edición facsimilar de la Academia Nacional de Medicina, México 1992.

2. Bennet, A.H., Goodle, R.J.: Exision of a tumor from the brain. Lancet. 1885; 2: 1090-1091.

3. Chico-Ponce de León, F., Diego Valadés: el funcionamiento del cerebro a las luces de la doctrina cavitaria. Primera imagen hecha por un americano. "Rhetorica christiana", Roma-Perugia, 1576. Salud Mental. 1999; 22: 29-36.

4. Chico-Ponce de León, F., Goodrich, J.T., Tutino, M., Gordon, C.: First published record of a neurosurgical procedure in the North American Continent, Mexico City, By Pedro Arias de Benavides, 1561: Secretos de Chirurgia, Valladolid, Spain, 1567. Neurosurgery. 2000; 47: 216-222.

5. Chico-Ponce de León, F., Boll-Woehrlen, M.C.: Guy de Chauliac et Alonso López de Hinojosos, Le premier livre de chirurgie du continent américain, Mexico, 1578. Histoire des Sciences Médicales 2000; XXXIV(3).

6. Chico-Ponce de León, F., Castro-Sierra, E.: The First Neuroanatomical Text Published in the American Continent: Mexico City, 1579. Childs Nerv Syst. 2004; 20: 8-17.

7. Chico-Ponce de León, F., Castro-Sierra, E., Goodrich, J.T.: Techniques of cranial surgery \& neuroanatomy in Mexico City, XVI Century. Lab. Bioquimed. Mexico, 2004; 124 pp.

8. Chico-Ponce de León, F.: El doctor Rafael Lavista y las primeras intervenciones sobre tumores cerebrales y cirugía de la epilepsia en México 1892. Arch Neurocien 2004; 9: 226232.

9. Chico-Ponce de León, F.: Historia de la cirugía de tumores cerebrales. En Aguirre-Cruz, Ma. Lucinda y Julio Sotelo Morales, eds. Tumores Cerebrales, Editorial Médica Panamericana, México 2008, pp. 313, p.1-16.

10. Dandy, W.E.: Surgery of the brain. In Lewis's Practice of Surgery. Hagerstown: WF Prior Co. 1932; 12: 1-682.

11. Dominguez-Galván, M.: Algunas consideraciones acerca de la trepanación oportuna en los tumores del cerebro. Tesis, para el examen general de Medicina, Cirugía y Obstetricia, Escuela Nacional de Medicina de México, Oficina tipográfica de la Secretaria de Fomento, México 1894.

12. Egea, R.: Herida de cabeza en la región frontal; fractura y hundimiento del hueso; trepanación sobre el seno longitudinal superior; curación. Gaceta Médica de México, tomo 14,1879 .

13. Farfán, A.: Tractado breve de anothomia y chirurgia... México, En casa de Antonio Ricardo, México, 1579. (Fotocopia de la Biblioteca "Nicolas León", de la Universidad Nacional Autónoma de México, México, 2001).
14. Fernández-Del Castillo, F.: Historia de la Academia Nacional de Medicina de México. Editorial Fournier, México, 1956.

15. Fernández-Del Castillo, F.; Bibliografía General de la Academia Nacional de Medicina. 1836-1956. Ediciones de la Academia Nacional de Medicina de México. México, 1959.

16. Flores y Troncoso, F. de A.: Historia de la Medicina en México. Oficina tipográfica de la Secretaría de Fomento, México 1886. Edición facsimilar del Instituto Mexicano del Seguro Social, 3 volúmenes, México, 1982.

17. Fuentes, M.: Nota Clínica sobre un caso de meduloblastoma con síndrome adiposo genital. Arch. De Neurol. Y Psiq. de México. 1938; 1-5: 296-313.

18. Fuentes, M., Vázquez, M.: Un caso de tumor del tercer ventrículo. Arch. De Neurol. Y Psiq. de México. 1939; 3: 493504.

19. Fuentes, M.: Tumor del tercer ventrículo ( $3^{\text {er }}$ caso). Arch. De Neurol. Y Psiq. de México. 1940; 3: 717-725.

20. Garay, A. de: Algunas palabras sobre cirugía cerebral. La Escuela de Medicina. 1891: XI; 290-295.

21. García Icazbalceta, J.: Bibliografía mexicana del Siglo XVI. Ed. Millares-Carlo A. Editorial del Fondo de Cultura Económica, Colección Biblioteca Americana, Mexico, 1954.

22. Goodrich, J.T.: Pediatric Neuro-Oncology. A Historical Perspective. In Tumors of the Pediatric Central Nervous System. Ed. Keating RF, Goodrich JT, Packer RJ. Thieme, New York, 2001.

23. Guerra, F.: Bibliografía Médica Americana y Filipina, dos volúmenes, Ollero \& Ramos Editores, Madrid 1999.

24. Guerra, F.: Medical colonization of the New World. Med Hist 1963; 7: 147-154.

25. Guevara-Oropeza, M.: Tumor del ángulo pontocerebeloso. Archivos de Neurología y Psiquiatría de México, 1938.

26. Hidalgo y Carpio, L.: Heridas del cráneo. Gaceta Médica de México. 1864; I: 33-46.

27. Hidalgo y Carpio, L.: Heridas de cráneo. Gaceta Médica de México. 1864; I: 349-351.

28. Hinojosa, P.: Herida de la cabeza curada por la operación del trépano. Unión Médica de México, órgano de la Academia de Medicina. 1856-1858; 2: 133.

29. Hurtado, F.: Reseña histórica de la cirugía del sistema nervioso. Gaceta Médica de México. 1914; 9: 307-311.

30. Icaza, R.: Un caso de epilepsia jacksoniana curado por la trepanación. Gaceta Médica de México. 1910; 5: 21.

31. Lavista, R.: Cirugía. Observación de un osteosarcoma del maxilar inferior, curado por la resección de la porción horizontal izquierda de dicho hueso. Gaceta Médica de México. 1864-1865; I: 230-232.

32. Lavista, R.: Nécesité et urgence de l'intervention opératoire dans les cas de lesiones cérébrales. Revista de Anatomía y Patología Clínicas. 1897; II: 550-557.

33. Lavista, R.: Informe que rinde al C. Ministro de Ins- 
trucción Pública, de las labores ejecutadas en el Museo de Anatomía Patológica, desde su fundación hasta la fecha, y Proyecto de reformas para su transformación en Instituto Anatomo-Patológico. Revista de Anatomía y Patología Clínicas. 1899: IV: 221-280.

34. León, N.: Los precursores de la literatura médica mexicana en los siglos XVI, XVII, XVIII y primer tercio del siglo XIX (hasta 1883). Gaceta Médica de México, Órgano de la Academia Nacional de Medicina. 1915; 10: 3-94.

35. León, N.: Apuntes para la historia de la enseñanza y el ejercicio de la Medicina en México desde la Conquista hispana hasta el año de 1833. Gaceta Médica de México, Órgano de la Academia Nacional de Medicina. 1915; 10: 466-489.

36. León, N.: Apuntes para la historia de la enseñanza y el ejercicio de la Medicina en México desde la Conquista hispana hasta el año de $18332^{\mathrm{a}}$ parte. Gaceta Médica de México, Órgano de la Academia Nacional de Medicina. 1916; 11: 210285.

37. León, N.: Apuntes para la historia de la enseñanza y el ejercicio de la Medicina en México desde la Conquista hispana hasta el año de $18333^{\mathrm{a}}$ parte. Gaceta Médica de México, Órgano de la Academia Nacional de Medicina. 1921; 2: 3-48.

38. León, N.: Datos para la historia de la medicina en México. Gaceta Médica de México, Órgano de la Academia Nacional de Medicina. 1925; 56: 12-30.

39. López de Hinojosos, A.: Summa y recopilacion de chirurgia, con un arte para sangrar muy util y provechosa. Antonio Ricardo, México, 1578. Edición de la Academia Nacional de Medicina, México, 1977.

40. López-Espinoza, J.A.: La primera revista médica de América. ACIMED 2000; 8: 133-139.

41. López-Piñeiro, J.M., García-Ballester, L.: El Renacimiento, siglo de oro de la cirugía española. In La trepanación en España. Ed López-Piñeiro JM. Clásicos neuroquirúrgicos españoles, Editorial Técnica Española, Madrid, 1967.

42. López-Piñeiro, J.M.: Los comienzos en España de la moderna neurocirugía durante los últimos años del siglo XIX y los primeros del XX. Ed López-Piñeiro JM. Clásicos neuroquirúrgicos españoles, Editorial Técnica Española, Madrid, 1967.

43. Marín, F.: Herida de la cabeza por arma de fuego. Gaceta Médica de México. Órgano de la Academia Nacional de Medicina. 1885; 20: 445-461.

44. de Martel, T., Rochard, M.E.: La Chirurgie craniene sous anesthésie locale. Bull et mém Soc nat de chir. 1918; 44 : 1364-1372.

45. Montes de Oca, F.: Herida penetrante del cráneo. Extracción del cuerpo extraño. Accidentes consecutivos. Muerte del enfermo. Autopsia. Gaceta Médica de México. 1870; 5: 253-260.

46. Mortera y Franyuti, B.: Algunas consideraciones sobre la intervención quirúrgica en el tratamiento de los tumores intra-craneanos. Tesis, para el examen general de
Medicina,Cirugía y Obstetricia, Escuela Nacional de Medicina de México, en la Imprenta del Gobierno Federal, en el ex-Arzobispado, México 1892.

47. Muriel, J.: Los Hospitales de la Nueva España. Universidad Nacional Autónoma de México y Cruz Roja Mexicana. México 1990.

48. Núñez, T.: Breves consideraciones sobre la operación del trépano, bajo el punto de vista de sus resultados y sobre una complicación que suele hacer necesaria la repetición de esta operación. Periódico de la Academia Nacional de Medicina de México. Tipografía y Litografía de Juan Aguilar Vera y Compañía. México 1908; VIII: 358-352.

49. Otero, M.: Descripción de un caso de fractura de cráneo con hundimiento, salvado por la trepanación. Revista La Escuela de Medicina. 1899; XV: 72-74.

50. Pagenstecher, G.: Rreflexiones sobre la trepanación y sus indicaciones en las afecciones traumáticas. Revista La Escuela de Medicina. 1899; XV: 46-54.

51. Pavón-Abreu, J.G.: Tumores del Angulo PontoCerebeloso. Dos casos Clínicos. Revista Mexicana de Psiquiatría, Neurología y Medicina Legal. 1942; (VIII) 48: 7-20.

52. Ramírez-Moreno, S.: Osteoma Intra-Craneano del Temporal Izquierdo. Revista Mexicana de Psquiatría, Neurología y Medicina Legal. 1938; (V) 29: 19-22.

53. Robles-Castillo, C.: Consideraciones al respecto de algunos casos de tumor cerebral. Gaceta Médica de México. 1940; 70: 241-333.

54. Robles-Castillo, C.: Quiste coloide del tercer ventrículo. Comunicación de un caso curado quirúrgicamente. Gaceta Médica de México. 1943; 74: 22-34.

55. Robles-Castillo, C.: Consideraciones acerca de cien casos de tumor cerebral. Gaceta Médica de México. 1944; 74 : 503-529.

56. Romo-Bolan, H.: Meningeoblastoma terebrante izquierdo. Revista Mexicana de Psquiatría, Neurología y Medicina Legal. 1941; (VII) 41: 33-36.

57. Somolinos-D'Ardois, G.: La medicina en las culturas mesoamericanas anteriores a la Conquista. (I), Capítulos de historia médica mexicana, Ed. Sociedad Mexicana de Historia y Filosofía de la Medicina, México, 1978.

58. Somolinos-D'Ardois, G.: El fenómeno de fusión cultural y su trascendencia médica (II), Capítulos de historia médica mexicana, Ed. Sociedad Mexicana de Historia y Filosofía de la Medicina, México, 1978.

59. Somolinos-D'Ardois, G.: Relación alfabética de los profesionistas médicos o en conexión con la medicina, que practicaron en territorio mexicano (1521-1618) (III). In Capítulos de historia médica mexicana. Ed. Sociedad Mexicana de Historia y Filosofía de la Medicina, México, 1978.

60. Somolinos-D'Ardois, G.: Relación y estudio de los impresos médicos mexicanos redactados y editados desde 1521 a 1618 (IV). In Capítulos de historia médica mexicana. Ed. Sociedad Mexicana de Historia y Filosofía de la Medicina, 
México, 1978.

61. Somolinos-D’Ardois, G.: La fusión indoeuropea en la medicina mexicana del Siglo XVI. In Medicina novohispana del Siglo XVI, Historia general de la medicina en México, Tomo II. Ed. Aguirre-Beltrán G, Moreno-de los Arcos R. Academia Nacional de Medicina / Universidad Nacional Autónoma de México. México, 1990.

62. Somolinos-D’Ardois, G.: Médicos y libros en el primer siglo de la colonia. In Medicina novohispana del Siglo XVI, Historia general de la medicina en México, Tomo II. Ed. Aguirre-Beltrán G, Moreno-de los Arcos R. Academia Nacional de Medicina / Universidad Nacional Autónoma de México. México, 1990.

63. Terán, J.M., Hidalgo y Carpio, L.: Observación de una herida de la cabeza con fractura, y hundimiento de esquirlas en la sustancia cerebral. Periódico de la Academia de Medicina de Megico. Segunda Época. 1843; I: 237-243.

64. Troconis-Alcalá, L.: Un caso de neoplasma maligno heterotípico de la fosa temporal izquierda. Gaceta Médica de México. 1911; 7: 391-395.

65. Urrutia, A.: Algunos aspectos de la cirugía cerebral. Periódico de la Academia Nacional de Medicina de México. Tipografía y Litografía de Juan Aguilar Vera y Compañía. Tomo IV, México 1904.

66. Velasco, M., Sanen, J.A.: Tumor del Hemisferio Izquierdo del Cerebelo. Revista Mexicana de Psquiatría, Neurología y Medicina Legal. 1938; (V) 29: 25-39.

67. Velasco-Suárez, M., Bautista-Martínez, J., García-
Oliveros, R., Weinstein, P.R.: Archaelogical origins of cranial surgery: Trephination in Mexico. Neurosurgery. 1992; 31: 3313-3319.

68. Walker, A.E.: A history of neurological surgery. The Williams and Wilkins Company, Philadelphia, 1951.

69.- Yaşargyl MG. Microneurosurgery. Volume IV A. CNS Tumors, Surgical Anatomy, Neuropathology, Neuroradiology, Neurophysiology, Clinical Considerations, Operability, Treatment Options. Georg Thieme Verlag Stutgart, 1994.

70. Yaşargyl, M.G.: Microneurosurgery. Volume IV B. CNS Tumors, Instrumentation and Equipement, Laboratory, Training, Surgical Approaches, Strategies, Tactics an Techniques, Surgery and Results of Extrinsic and Intrinsic Tumors, Interventional Neuroradiology, Neuroanesthesia, complications. Georg Thieme Verlag Stutgart, 1996.

71. Zarraga, F.: Un caso de tumor cerebral. La Escuela de Medicina, 1891; XI: 38-400.

72. Zuckermann, C.: Concepto Anatomo-Patológico de los tumores cerebrales. Revista Mexicana de Psiquiatría, Neurología y Medicina Legal. 1935; (II) 9: 29-32.

73. Zuckermann, C.: Estado Actual de la Cirugía de tumores cerebrales. Revista Mexicana de Psiquiatría, Neurología y Medicina Legal. 1939; (VI) 33: 5-38.

Chico Ponce de León, F.: Historia de la cirugía de cráneo, de los tumores cerebrales y de la epilepsia en Mexico. Neurocirugía 2009; 20: 388-399. 